\title{
Бериллий как материал для термостойких рентгеновских зеркал
}

\author{
(C) Н.И. Чхало ${ }^{1}$, М.В. Зорина ${ }^{1}$, И.В. Малышев ${ }^{1}$, А.Е. Пестов ${ }^{1}$, В.Н. Полковников ${ }^{1}$, Н.Н. Салащенко $^{1}$, \\ Д.С. Казаков ${ }^{2}$, А.В. Мильков ${ }^{2}$, И.Л. Струля ${ }^{2}$ \\ ${ }^{1}$ Институт фризики микроструктур РАН, \\ 607680 Нижний Новгород, Россия \\ ${ }^{2} \mathrm{OAO}$ „Композит“, \\ 141070 Королев, Московская обл., Россия \\ e-mail: chkhalo@ipmras.ru
}

Поступило в Редакцию 28 марта 2019 г.

В окончательной редакции 28 марта 2019 г.

Принято к публикации 15 апреля 2019 г.

Проведено сравнение теплофизических и механических характеристик бериллия с рядом наиболее перспективных материалов, применяемых для изготовления высокоточных зеркал, работающих в условиях сильных потоков электромагнитного излучения. Обсуждены достоинства бериллия и перспективы его применения для синхротронов 3-го и 4-го поколения. Сообщено о разработанной авторами методике получения сверхгладких поверхностей бериллиевых подложек и полученных предельных шероховатостях. Приведены данные по коэффициентам отражения на длине волны $13.5 \mathrm{~nm}$ многослойного Mo/Si-зеркала, нанесенного на его поверхность. Обсуждены перспективы повышения качества полировки бериллиевых подложек.

Ключевые слова: бериллий, синхротронное излучение, зеркало, рентгеновская оптика, шероховатость, поверхность, искажения формы.

DOI: $10.21883 /$ JTF.2019.11.48329.134-19

\section{Введение}

Экстремально большие, до киловатта и выше, потоки синхротронного излучения источников 3-го и 4-го поколения и лазеров на свободных электронах приводят к заметным градиентам температур в первых рентгенооптических элементах. В свою очередь температурные градиенты искажают форму поверхности зеркал и атомную решетку кристаллов. Все это приводит к деградации свойств отраженных пучков. Поэтому проблема минимизации температурных градиентов в первых оптических элементах является ключевой задачей для разработчиков. Проблема решается за счет выбора оптимальных материалов и оптимизации системы охлаждения зеркал, в частности, использования охлаждающих каналов различной формы внутри элементов [1]. В качестве материала подложек зеркал используется кремний, охлаждаемый как жидким азотом, так и водой [2,3]. Реже применяется карбид кремния и медь [4]. В работе [5] предлагалось алюминиевое зеркало с оксидным слоем, сформированным методом микроплазменного оксидирования. Бесспорным лидером является кремний.

Проблема термоиндуцированных искажений зеркал для синхротронов 3-го, 4-го поколения осложняется малым размером источника излучения, на уровне $10 \mu \mathrm{m}$, и для сохранения его когерентных свойств угловые ошибки зеркал должны лежать в субмикрорадианном диапазоне, на уровне $0.1 \mu \mathrm{rad}$ [6]. Поэтому для обеспечения размерной стабильности зеркал, помимо высоких теплофизических характеристик материала, боль- шое значение приобретают и его механические характеристики, обеспечивающие возможность оптимизации конструкции подложки для повышения эффективности охлаждающей системы и меньшие искажения при различных механических воздействиях, например, при установке зеркала на канале, сопряжении зеркала и системы охлаждения и др.

Бериллий, обладая уникальным сочетанием механических характеристик, прежде всего низкой плотностью и высокой жесткостью [7], уже десятки лет используется при изготовлении конструкционных материалов и зеркал на космических аппаратах [8-11]. Из последних разработок особо следует выделить телескоп James Webb Telescope с диаметром первичного зеркала $6.5 \mathrm{~m}$, который должен прийти на смену всемирно известному телескопу Hubble Space Telescope [12]. Несмотря на широкое применение в оптическом производстве бериллий ранее никем не рассматривался в качестве подложек для рентгеновской оптики и, в частности, в качестве материала для высокоточных зеркал, работающих в условиях облучения потоками рентгеновского излучения с мощностью от 1 до $n \cdot 10 \mathrm{~kW}$.

В настоящей работе проводится сравнение теплофизических и механических характеристик бериллия с рядом наиболее перспективных материалов, применяемых для изготовления высокоточных зеркал, работающих в условиях сильных потоков электромагнитного излучения. Обсуждаются достоинства бериллия и перспективы его применения для синхротронов 3-го и 4-го поколения. Сообщается о разработанной авторами методике получе- 
Наиболее перспективные материалы для зеркал в сравнении с бериллием

\begin{tabular}{l|c|c|c|c|c|c}
\hline \multicolumn{1}{c|}{ Материал } & $\begin{array}{c}\gamma, \\
\mathrm{W} /(\mathrm{cm} \cdot \mathrm{K})\end{array}$ & $\begin{array}{c}\alpha, \\
\mathrm{K}^{-1} \cdot 10^{-6}\end{array}$ & $\begin{array}{c}E, \\
\mathrm{GPa}\end{array}$ & $\begin{array}{c}\rho \\
\mathrm{g} / \mathrm{cm}^{3}\end{array}$ & $\begin{array}{c}\alpha / \gamma, \\
\mathrm{m} / \mathrm{W} \cdot 10^{-6}\end{array}$ & $\begin{array}{c}E / \rho, \\
\mathrm{GPa} \cdot \mathrm{cm}^{3} / \mathrm{g}\end{array}$ \\
\hline Кремний монокристалл & 1.53 & 2.10 & 135 & 2.33 & 1.37 & 57.9 \\
Карбид кремния поликристаллический & 0.31 & 2.45 & 400 & 3.22 & 7.90 & 124 \\
Оптическая керамика ULE & 0.013 & 0.03 & 68 & 2.21 & 2.3 & 30.8 \\
Медь & 4.01 & 16.7 & 120 & 8.93 & 4.16 & 13.4 \\
Алюминий & 2.37 & 23.3 & 70.5 & 2.69 & 9.83 & 29.7 \\
Бериллий & 2.10 & 11.2 & 300 & 1.85 & 5.33 & 162
\end{tabular}

ния сверхгладких поверхностей бериллиевых подложек и полученных предельных шероховатостях. Приводятся данные по коэффициентам отражения на длине волны $13.5 \mathrm{~nm}$ многослойного Mo/Si-зеркала, нанесенного на его поверхность. Обсуждаются перспективы повышения качества полировки бериллиевых подложек.

\section{Критерии выбора и сравнение перспективных материалов для термостойких высокоточных зеркал с Be}

При практическом использовании высокоточных зеркал необходимо учитывать ряд факторов, влияющих на качество отраженных от них фронтов. Из анализа этих факторов вырабатываются критерии выбора того или иного материала для зеркал. Одним из факторов являются механические напряжения, возникающие при установке зеркал в оправы или их закрепление в приборах. Из-за этих напряжений происходит деформация поверхности $\Delta_{m s}$, которая может достичь неприемлемой величины. Согласно закону Гука, величина этой деформации обратно пропорциональна модулю Юнга $E$

$$
\left(\Delta_{m s} \propto E^{-1}\right) .
$$

Из этого соотношения следует, что чем выше модуль Юнга материала, тем он имеет лучшую размерную стабильность по отношению к механическим воздействиям.

Другим фактором является деформация поверхности под действием силы тяжести Земли. Соответствующая деформация $\Delta_{g r}$ зависит от удельной жесткости согласно соотношению:

$$
\left(\Delta_{m s} \propto \rho E^{-1}\right),
$$

где $\rho-$ плотность материала. Гравитационные искажения будут тем меньше, чем меньше плотность и выше модуль Юнга материала.

В прецизионных оптических системах часто разрешение ограничивается вибрациями оптических элементов. Известно, что традиционные системы подавления вибраций лучше работают на относительно высоких $(\gg 10 \mathrm{~Hz})$ частотах и плохо - на низких. Собственная частота колебаний зеркала $f_{r}$ зависит от механических характеристик материала как

$$
f_{r} \propto(E / \rho)^{1 / 2}
$$

И снова мы видим, что чем выше модуль Юнга и меньше плотность, тем предпочти- тельнее материал.

Для зеркал, эксплуатируемых в условиях сильных тепловых нагрузок, критическим фактором являются термоиндуцированные деформации формы поверхности. Величина деформации $\Delta_{t}$ зависит от коэффициентов теплового расширения $\alpha$ и теплопроводности $\gamma$, согласно соотношению, например [1]:

$$
\Delta_{t} \propto \alpha / \gamma
$$

Как следует из (4), чем меньше коэффициент температурного расширения и выше теплопроводность, тем меньше деформации. В таблице приведены параметры наиболее перспективных кандидатов для зеркал в сравнении с бериллием. Механические и теплофизические характеристики материалов за исключением оптической керамики ULE были взяты из [7]. Необходимо отметить, что приведенные значения основных характеристик материалов сравнивались с данными других работ и находятся в хорошем согласии. Исключение составляет карбид кремния. Это связано с тем, что в ряде работ приводится существенно больший коэффициент теплопроводности $\gamma$, который наблюдается для слоев, полученных методов CVD (chemical vapor deposition). B реальных зеркалах этот слой достаточно тонок и основные теплофизические характеристики определяются все же основным телом зеркала, состоящее из поликристаллического карбида кремния. Параметры ULE взяты из [13].

Как видно из таблицы, с точки зрения тепловых деформаций на первом месте стоит кремний. Поэтому параметру совсем немного уступает ULE - оптическая керамика с экстремально низким коэффициентом линейного расширения. Однако это диэлектрик, и он не может использоваться под мощными пучками синхротронного излучения. Бериллий уступает кремнию по этому параметру почти в 4 раза и находится на уровне меди. По механическим характеристикам Ве наиболее предпочтителен из всех рассматриваемых материалов, уступая только по модулю Юнга SiC. Однако и в этом случае по удельной жесткости Ве превосходит $\mathrm{SiC}$. 


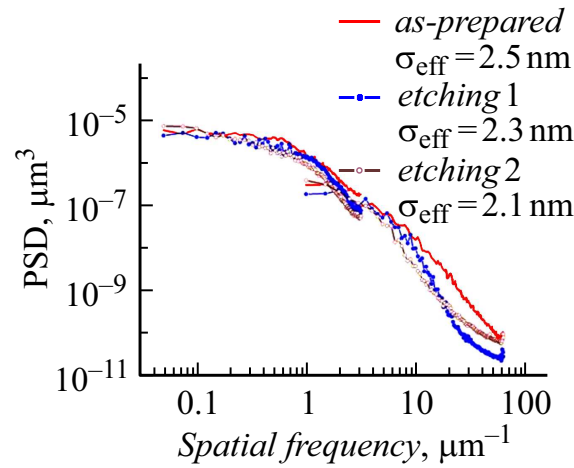

$b$

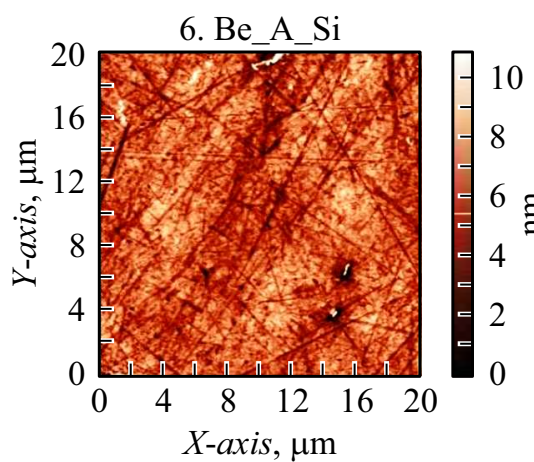

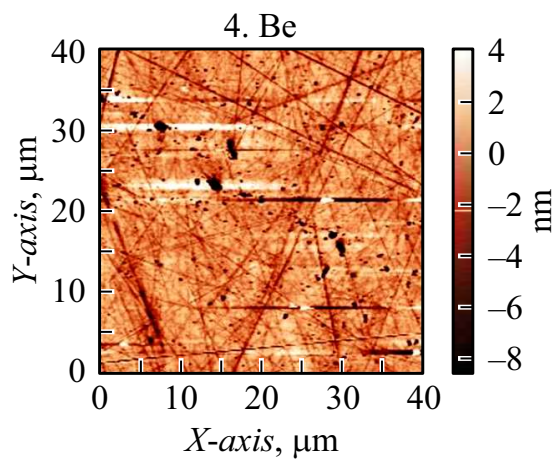

Рис. 1. Функции спектральной плотности мощности шероховатостей бериллиевой подложки после полировки и после последующих двух процедур травления ионами аргона с энергией $400 \mathrm{eV}(a)$ и $\mathrm{ACM}$ изображения поверхности, кадры $40 \times 40 \mu \mathrm{m}$, после полировки $(b)$ и после 2-го травления ионами $(c)$.

Несмотря на то что бериллий уступает кремнию по коэффициенту тепловых деформаций $\alpha / \gamma$, по своим теплофизическим характеристикам он может даже превзойти кремний. Это связано со следующими обстоятельствами. Во-первых, благодаря лучшей теплопроводности при прочих равных условиях у зеркал из Ве будет ниже максимальная температура, что особенно важно для синхротронов 4-го поколения. Во-вторых, как было показано в ряде работ и теоретически, и экспериментально, помимо теплофизических характеристик материала для высокоточных зеркал, находящихся под воздействием мощных потоков излучения, ключевое значение приобретает эффективность системы охлаждения. Поэтому выражение (4) должно быть помножено на коэффициент $G$, который зависит от способа охлаждения зеркала. При этом величина этого коэффициента может изменяться в пределах 0.01-1 [14]. Иными словами, фактор $G$ является ключевым при выборе материала. Так как способ охлаждения зависит от конструкции зеркала, которая, в свою очередь, определяется уже механическими характеристиками материала и возможностью его обработки на станках, то в этом отношении бериллий существенно превосходит кремний. В частности, в [3] подробно анализируются деформации зеркал в зависимости от способа их охлаждения при одинаковых радиационных нагрузках $840 \mathrm{~W}$ и эффективности теплосъема $0.005 \mathrm{~W} / \mathrm{mm}^{2} /{ }^{\circ} \mathrm{C}$, характерных для Европейского синхротрона ESRF, Франция, относящегося к машинам 3-го поколения. В работе показано, что наличие дополнительных пазов в подложке существенно на порядок величины, меняет термоиндуцированную ошибку формы поверхности. На ошибку сильное влияние оказывают и форма и размеры пазов. С учетом сложности обработки кремния, и наоборот, - простоты бериллия, можно ожидать, что бериллий будет способен работать при еще больших тепловых нагрузках.

Подводя итог этому разделу можно заключить, с точки зрения механических и теплофизических характеристик бериллий является перспективным материалом для изготовления высокоточных зеркал для синхротронов 3-го и особенно 4-го поколения.

\section{Полируемость и формообразование бериллиевых подложек}

Другим важным свойством материала для рентгенооптических приложений является его полируемость, т. е. возможность придания поверхности заданной формы при низкой шероховатости отражающей поверхности. Из-за малой длины волны спецификой задачи являются более жесткие требования к шероховатости, на уровне $n \cdot 0.1 \mathrm{~nm}$, и точности формы, nm. Как правило, для достижения нанометровой точности формы поверхности на финальной стадии изготовления подложек для зеркал используется ионно-пучковая коррекция локальных ошибок формы [15-17].

Хорошо известно, что для инфракрасного и видимого диапазонов достигнутый уровень полировки бериллия в полной мере удовлетворяет требованиям. Полировались как чисто бериллиевые подложки [11], так и покрытые электрохимическим никелем [18], и тонким слоем стекла со специально подобранным к бериллию коэффициентом температурного расширения [19]. Однако насколько достигнутые параметры поверхности соответствуют рентгенооптическим задачам, а также возможность ионнопучковой обработки бериллия ранее не изучались.

В настоящей работе изучались подложки чистого бериллия и покрытые электрохимическим никелем с аморфной по данным рентгеновской дифракции структурой. В качестве исходного материала выбран бериллий, технический спеченный марки ТГП с содержанием бериллия 97.8\%. Полировка производилась с применением традиционной оптической технологии. В качестве полирующего материала использовалась пеко-канифольная смола. Абразивная суспензия готовилась на основе 1\% раствора щавелевой кислоты с добавлением микропорошков синтетического алмаза. Последовательно приме- 

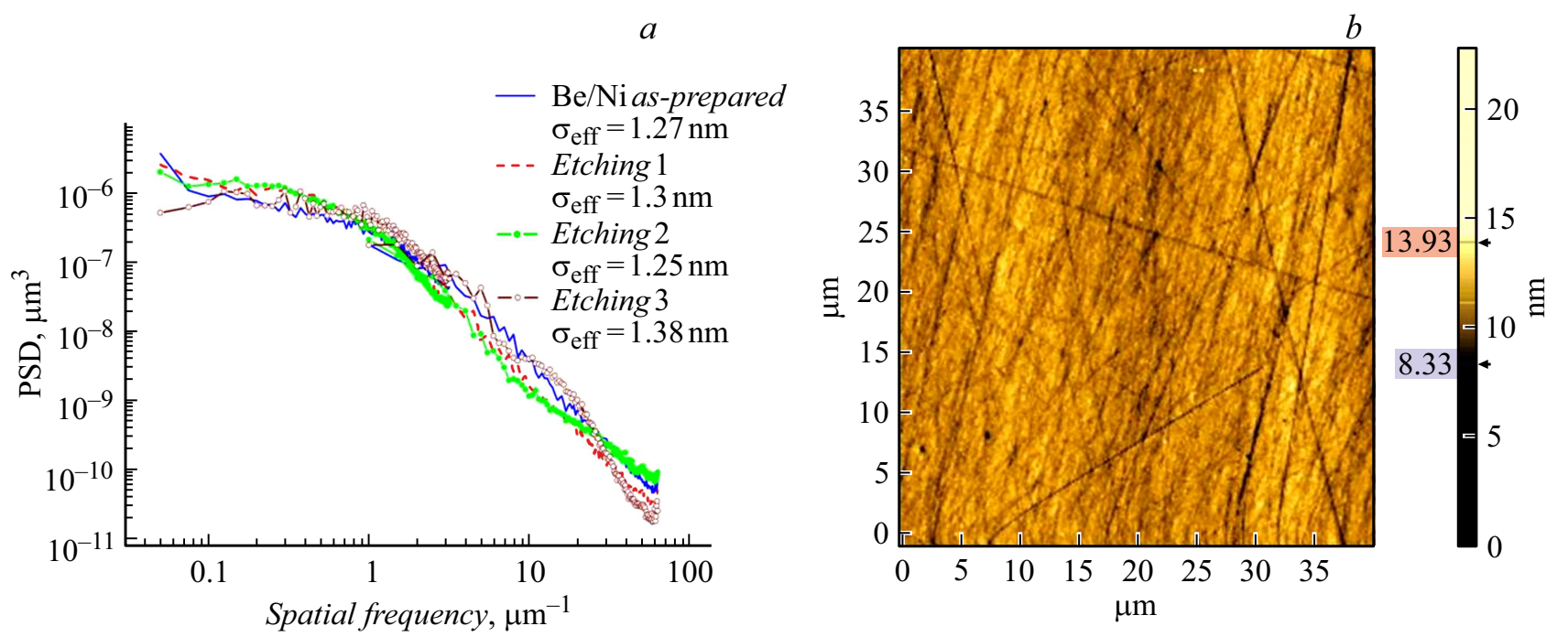

Рис. 2. PSD-функции шероховатостей после полировки и после трех процедур травления ионами аргона с энергией 300 , 400 и $800 \mathrm{eV}$ - (слева), и АCM изображение поверхности после травления ионами (справа). Подложка бериллий, покрытый никелем.

нялись микропорошки фракции $10 / 7,5 / 3,3 / 2$ и $1 / 0 \mu \mathrm{m}$. Дополнительная доводка на синтетических полирующих подложках не производилась.

На рис. 1 приведены функции спектральной плотности мощности шероховатостей (PSD-power spectral density) бериллиевой подложки после полировки, и после последующих двух процедур травления ионами аргона с энергией $400 \mathrm{eV}$ (рис. 1,a), АCM изображения поверхности, кадры $40 \times 40 \mu \mathrm{m}$, после полировки (рис. $1, b)$, после 2-го травления ионами (рис. 1,c). Методика измерений и обработки экспериментальных результатов подробно изложена в [20], эксперименты по ионному травлению были проведены на установке и с использованием методов, описанных в [21]. Максимальная глубина травления составила $300 \mathrm{~nm}$. Из этих данных следует, что после полировки эффективная шероховатость составила $2.5 \mathrm{~nm}$, что примерно на порядок хуже, чем у кремния. Травление ионами существенно не влияет на шероховатость поверхности, более того, даже наблюдается незначительное сглаживание. Эффективная шероховатость упала до $2.1 \mathrm{~nm}$. Однако на поверхности появляются ямы, которых не наблюдалось на исходной поверхности. Причиной появления ямок является неоднородность состава материала, наличие различного рода включений, примесей, имеющих отличные от массивного материала коэффициенты распыления.

На основе этого исследования можно сделать следующие выводы. Во-первых, качество полировки бериллия не достаточно для рентгенооптических приложений. Вовторых, материал не допускает обработку ионами. Причем, как показали исследования, эффект расстравливания поверхности слабо зависит от энергии ионов, по крайней мере, в диапазоне энергий $200-1000 \mathrm{eV}$.

Аналогичные исследования были проведены с бериллиевой подложкой, покрытой электролитическим нике-

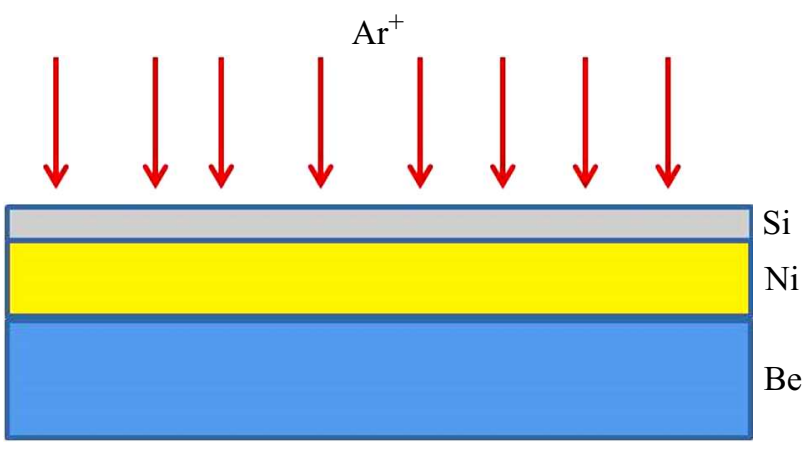

Рис. 3. Схема, поясняющая идею ионно-пучковой полировки $\mathrm{Be}:$ Ni-подложек.

лем. На рис. 2 для этой подложки приведены PSDфункции шероховатостей после полировки и после последующих трех процедур травления ионами аргона с энергией 300 (Etching 1), 400 (Etching 2) и 800 (Etching 3) eV (рис. 2,a), АСМ изображения поверхности после травления ионами (рис. 2, $b$ ). Глубина травления во всех экспериментах составила $250 \mathrm{~nm}$.

Из приведенных экспериментальных данных можно сделать следующие выводы. Во-первых, эффективная шероховатость упала почти в 2 раза, с 2.4 до $1.37 \mathrm{~nm}$ и, что самое важное, ионное травление не влияет ни на шероховатость, ни на морфологию поверхности. Это означает, что форму бериллиевых подложек, покрытых никелем, можно корректировать с помощью ионов, по крайней мере, до глубин съема материала $250 \mathrm{~nm}$ (характерный съем материала при ионно-пучковой коррекции формы). Во-вторых, эффективная шероховатость все же в 3-4 раза хуже требуемой для рентгенооптических приложений. 


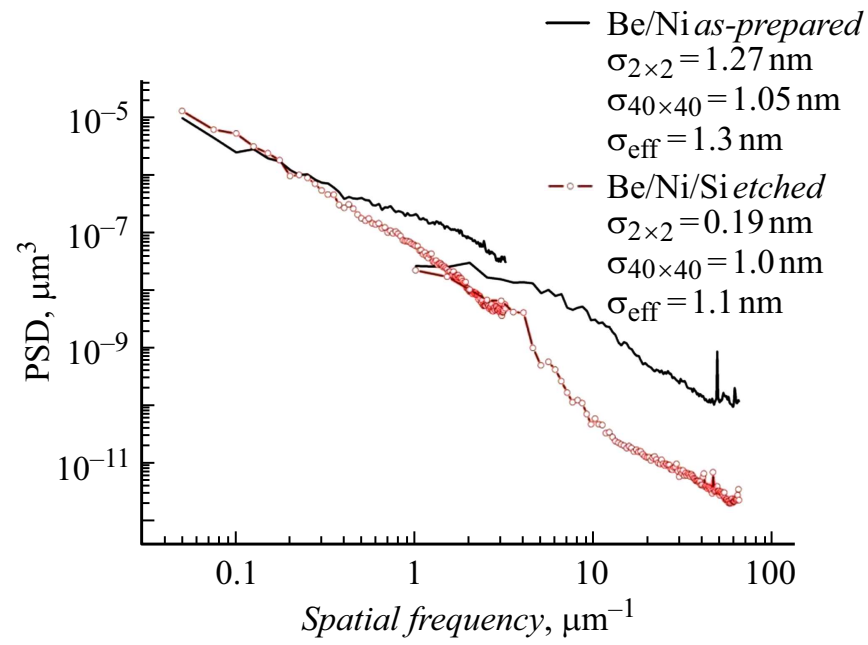

Рис. 4. PSD-функции шероховатостей для образца $\mathrm{Be}: \mathrm{Ni}: \mathrm{Si}$ после нанесения $\mathrm{Si}$ (верхняя кривая) и после травления (нижняя).

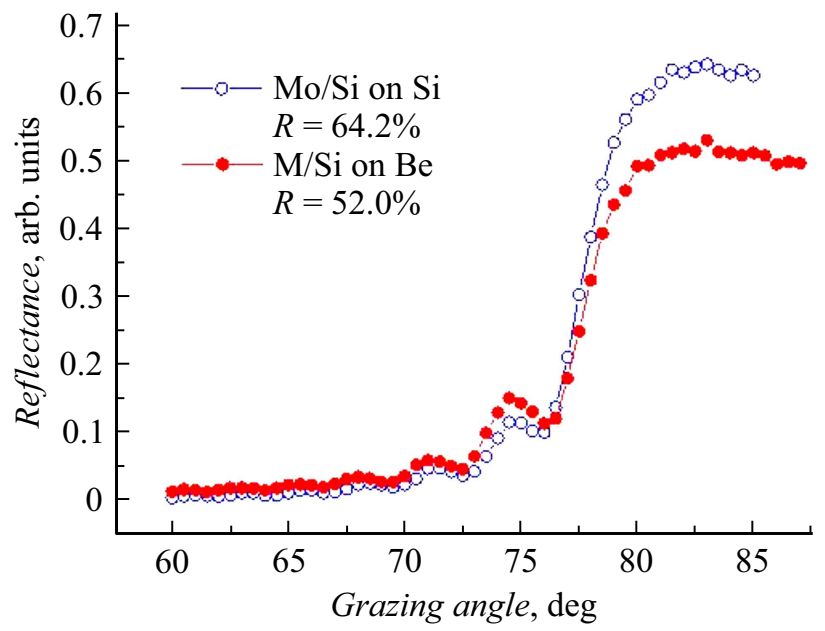

Рис. 5. Угловые зависимости коэффициента отражения на длине волны $13.5 \mathrm{~nm} \mathrm{Mo/Si} \mathrm{многослойных} \mathrm{зеркал,} \mathrm{нанесенных}$ на $\mathrm{Be}: \mathrm{Ni}: \mathrm{Si}-$ подложку после ионной полировки (нижняя кривая) и на суперполированную Si-подложку-свидетель (верхняя кривая).

В работе [22] мы нашли прием, позволивший нам существенно до ангстремного уровня улучшить шероховатость металлических пленок с помощью ионнопучкового травления. Рис. 3 поясняет идею. На $\mathrm{Be}: \mathrm{Ni}$ полированную подложку методом магнетронного распыления наносилось примерно $0.2 \mu \mathrm{m}$ аморфного кремния. Далее пленка полировалась ионами с энергией $800 \mathrm{eV}$.

На рис. 4 приведены PSD-функции шероховатостей для образца $\mathrm{Be}: \mathrm{Ni}: \mathrm{Si}$ после нанесения $\mathrm{Si}$ - верхняя кривая и после травления. Как уже отмечалось в большинстве работ при ионно-пучковом травлении ионами малых энергий и нормальном падении аморфных материалов происходит сглаживание мелкомасштабных (высокочастотных) шероховатостей, при этом средне- частотные не изменяются [23]. И в нашем случае мы видим резкое падение высокочастотных шероховатостей (размер АСМ кадра $2 \times 2 \mu \mathrm{m}$ ) с 0.46 до $0.16 \mathrm{~nm}$. К сожалению, на шероховатости с частотой меньше $0.5 \mu \mathrm{m}^{-1}$ ионное травление не оказывает заметного влияния, поэтому эффективная шероховатость уменьшилась незначительно, с $1.3 \mathrm{mn}$ до $1.1 \mathrm{~nm}$. Так как на величину коэффициента отражения в рентгеновской области в наибольшей степени влияют высокочастотные шероховатости, то можно было ожидать достаточно высоких коэффициентов отражения многослойных рентгеновских зеркал, нанесенных на эти подложки.

На рис. 5 приведены угловые зависимости коэффициента отражения на длине волны $13.5 \mathrm{~nm} \mathrm{Mo} / \mathrm{Si}$ многослойных зеркал, нанесенных на $\mathrm{Be}: \mathrm{Ni}$ : $\mathrm{Si}$-подложку после ионной полировки (нижняя кривая) и на суперполированную $\mathrm{Si}$-подложку-свидетель (верхняя кривая). Как видно из рисунка, при коэффициенте отражения на свидетеле $64 \%$, коэффициент отражения на бериллиевой подложке составил около $52 \%$, что уже вполне достаточно для практического использования. Для сравнения коэффициенты отражения $\mathrm{Mo} / \mathrm{Si}$ многослойных зеркал, нанесенных на подложки полированного Ве и $\mathrm{Be}: \mathrm{Ni}$, составили 1.5 и $28 \%$ соответственно. Таким образом, мы впервые показали возможность напыления многослойных высокоотражающих зеркал на бериллий.

\section{Обсуждение результатов и выводы}

Основными результатами работы стали следующие выводы.

1. Показано, что благодаря большей теплопроводности высоким механическим характеристикам и простоте механической обработки бериллий существенно расширяет окно возможностей для оптимизации системы охлаждения по сравнению с кремнием, тем самым, потенциально обеспечивая меньшие рабочие температуры и термоиндуцированные искажения формы зеркал. Особенно преимущества бериллия должны проявиться для синхротронов 4-го поколения, для которых характерны повышенные мощности пучков синхротронного излучения и где еще большее значение приобретает система охлаждения зеркал.

2. Показана возможность ионно-пучковой обработки бериллия с никелевым покрытием без ухудшения шероховатости и топологии поверхности. По аналогии c $[24,25]$ это свойство позволяет применять методику коррекции локальных ошибок формы поверхности зеркал с помощью ионно-пучкового травления, что, в конечном итоге, позволяет достигать нанометровых и даже субнанометровых точностей.

3. Разработана комплексная методика полирования бериллиевых подложек, включающая на финальной стадии ионно-пучковое травление аморфного кремния, нанесенного на поверхность бериллия, позволяет получать шероховатости, уже достаточные для решения некоторых 
рентгенооптических задач. Впервые на такие подложки были нанесены многослойные зеркала нормального падения с коэффициентами отражения, близкими к образцам, нанесенным на суперполированные кремниевые подложки.

4. Нерешенной на данный момент времени проблемой являются высокие среднечастотные шероховатости, которые примерно в 3 раза превышают предельно допустимые для рентгеновской оптики дифракционного качества. Однако из собственного опыта работ мы знаем, что для уменьшения среднечастотной шероховатости надо на финальной стадии механической полировки использовать более мелкие полирующие порошки. В настоящей работе механическая полировка заканчивалась стандартными порошками алмаза микронного размера. В работе [26] было показано, что при полировании плавленого кварца на финальной стадии при переходе с порошка с размером $1 \mu \mathrm{m}$ к порошку $0.2 \mu \mathrm{m}$ среднечастотная шероховатость упала более чем в 3 раза, что в конечном итоге, дополнив этот результат ионнопучковой полировкой ионами ксенона, удалось достичь рекордно низких шероховатостей. Мы надеемся, что этот подход позволит уменьшить среднечастотную ше-

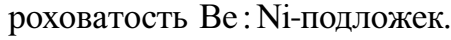

\section{Финансирование работы}

Работа выполнена в рамках государственного задания 0035-2014-0204, при поддержке грантов Российского фонда фундаментальных исследований 19-02-00081, 1802-00588, 18-02-00173, 18-07-00633, 18-32-00149 мол_а и 17-02-00640.

\section{Конфликт интересов}

Авторы заявляют, что у них нет конфликта интересов.

\section{Список литературы}

[1] Erko A., Idir M., Krist Th., Michette A.G. Modern Developments in X-ray and Neutron Optics. NY: Springer Berlin Heidelberg, 2008. 533 p. ISBN 978-3-540-74560-0

[2] Bilderback D.H., Freund A.K., Knapp G.S., Mills D.M. // J. Synchrotron Radiation. 2001. Vol. 8. P. 22.

[3] Zhang L., Barrett R., Friedrich K., Glatzel P., Mairs T., Marion P., Monacol G., Morawe1 C., Weng T. // J. Physics: Conference Series. 2013. Vol. 425. P. 052029. DOI: $10.1088 / 1742-6596 / 425 / 5 / 052029$

[4] Takacs P.Z. // Synchrotron Radiation News. 1989. Vol. 2. N 26. P. 24.

[5] Chkhalo N.I., Fedorchenko M.V., Kovalenko N.V., Kruglyakov E.P., Volokhov A.I., Chernov V.A., Mytnichenko S.V. // Nuclear Instruments and Methods in Physics Research. 1995. A. 359. P. $121-126$.

[6] Admans G., Berkvens P., Kaprolat A., Revol J.-L. ESRF upgrade programme phase II (2015-2022). technical design study: Imprimerie de Pont de Claix (C) ESRF, December 2014. 192 p. http://www.esrf.eu/Apache_files/Upgrade/ESRForange-book.pdf
[7] Физические величины: Справочник / Под ред. И.С. Григорьева, Е.3. Мейлихова. М.: Энергоатомиздат, 1991. 1232 с.

[8] Jr. Barnes W.P. // Appl. Opt. 1966. Vol. 5. N 12. P. 1883.

[9] Fanson J.L., Fazio G.G., Houck J.R., Kelly T., Rieke G.H., Tenerelli D.J., Whitten M. // Proc. SPIE, Space Telescopes and Instruments. Vol. 3356. P. 478. 1998.

[10] Gildner D.A., Marder J.M. // Proc. SPIE, Reflective and Refractive Optical Materials for Earth and Space Applications. 1991. P. 1485.

[11] Сизенев В.С., Струля И.Л., Григоревский А.В., Просвириков В.М., Менделеев В.Я., Сковородько С.Н. // ВАНТ. Серия: Физика радиационных повреждений и радиационное материаловедение. 2010. Т. 95. № 1. С. 21-27.

[12] Hashiguchi D., Marder J., Paquin R. // Advanced Materials \& Processes. 2015. Vol. 173. N 8. P. 20-27.

[13] Gulati S.T., Edwards M.J. // Proc. SPIE 10289, Advanced Materials for Optics and Precision Structures: A Critical Review. 1997. P. 1028909. DOI: $10.1117 / 12.284710$

[14] Barrett R. X-ray Optics for Synchrotron Radiation Beamlines. ESI 2011: Grenoble, France, 15-22 May 2011. http://www.epn-campus.eu/fileadmin/_migrated/content_uploads/16.BARRETT_XRayOptics.pdf

[15] Weiser M. // Nucl. Instrum. Method. Phys. Res. Sect. 2009. B. 267. P. $1390-1393$.

[16] Chkhalo N.I., Kluenkov E.B., Pestov A.E., Polkovnikov V.N., Raskin D.G., Salashchenko N.N., Suslov L.A., Toropov M.N. // Nucl. Instrum. Method. Phys. Res. A. 2009. Vol. 603. N 1-2. P. 62-65.

[17] Arnold T., Bohm G., Fechner R., Meister J., Nickel A., Frost F., Hansel T., Schindler A. // Nucl. Instrum. Method. Phys. Res. Sect. 2010. A 616. P. 147-156.

[18] Campbell J.C. // Proc. SPIE, Design, Manufacture and Application of Metal Optics. 1976. Vol. 97. P. 0065.

[19] Мирошников М.М., Любарский С.В., Химич Ю.П. // Зеркала оптических телескопов. Оптико-механическая промышленность. 1990. № 9. С. 3-18.

[20] Barysheva M.M., Vainer Yu.A., Gribkov B.A., Zorina M.V., Pestov A.E., Rogachev D.N., Salashenko N.N, Chkhalo N.I. // Bulletin of the Russian Academy of Sciences: Physics. 2011. Vol. 75. N 1. P. 67-72.

[21] Chkhalo N.I., Kaskov I.A., Malyshev I.V., Mikhaylenko M.S., Pestov A.E., Polkovnikov V.N., Salashchenko N.N., Toropov M.N., Zabrodin I.G. // Precision Engineering. 2017. Vol. 48. P. 338-346

[22] Chkhalo N.I., Mikhailenko M.S., Mil'kov A.V., Pestov A.E., Polkovnikov V.N., Salashchenko N.N., Strulya I.L., Zorina M.V., Zuev S.Yu. // Surf. Coatings Technol. 2017. Vol. 311. P. 351.

[23] Chkhalo N.I., Churin S.A., Mikhaylenko M.S., Pestov A.E., Polkovnikov V.N., Salashchenko N.N., Zorina M.V. // Appl. Opt. 2016. Vol. 55. N 6. P. 1249-1256.

[24] Chkhalo N.I., Malyshev I.V., Pestov A.E., Polkovnikov V.N., Salashchenko N.N., Toropov M.N., Soloviev A.A. // Appl. Opt. 2016. Vol. 55. N 3. P. 619-625.

[25] Электронный ресурс. Режим доступа: http://seso.com/wpcontent/themes/theme44938/images/PDF/Brochure/optimise/ BROCHURE\%20-\%20X-RAY.pdf

[26] Chkhalo N.I., Churin S.A., Pestov A.E., Salashchenko N.N., Vainer Yu.A., Zorina M.V. // Opt. Expres. 2014. Vol. 22. N 17. P. 20094-20106. 